\title{
Terminal de Acesso de Baixo Custo para Ensino Remoto
}

DOI: 10.37702/2175-957X.COBENGE.2021.3751

Diego Tefili - diego.tefili@ifpr.edu.br Instituto Federal do Paraná

Rua Dep Joaquim José Pedrosa 373

80025-120 - Curitiba - PR

Beatriz dos Santos Pês - beatriz.santos@ifpr.edu.br

Instituto Federal do Paraná

Rua Monsenhor Ivo Zanlorenzi 1759

81210-000 - Curitiba - PR

Marcos Santos Hara - mshara@gmx.de

Instituto Federal do Paraná

Rua Herculano Carlos Franco de Souza 628

80240-290 - Curitiba - PR

Gabriel Schwartz Scarpin - gabrielsscarpin@gmail.com

Instituto Federal do Paraná

joaquim cicarino norberto 194

83602-135 - Campo Largo - PR

Eduardo Jean Falarz - eduardofalarz23@gmail.com

Instituto Federal do Paraná

Rua João Cosmo 487

83607-130 - Campo Largo - PR

Alexandre Padilha - alexpadilha2083@gmail.com

Instituto Federal do Paraná

Rua Visconde de Taunay 341

83606-230 - Campo Largo - PR

Resumo: Devido à suspensão de aulas presenciais de 2020 no Brasil, alternativas digitais foram adotadas para possibilitar algum tipo de ensino remoto. Uma das formas de comunicação que se popularizou foi a videoconferência. O presente projeto visa oferecer uma solução a uma das dificuldades das atividades remotas: 
a falta de um dispositivo adequado ao estudo. Para isso, utilizou-se um kit composto de um computador de placa única de tamanho reduzido com os periféricos necessários para a participação em aulas remotas e execução das atividades didáticas. $O$ kit foi preparado para ser usado como terminal de acesso à internet por alunos que não dispõem de computador. Este kit pode ser conectado a uma televisão com entrada HDMI. Dentre as vantagens do desenvolvimento deste kit cita-se o baixo custo da proposta, quando comparado ao custo de computadores comerciais. Diferentes placas e sistemas operacionais foram testados, obtendo-se resultados satisfatórios de navegação na internet $e$ participação em chamadas de vídeo com a placa Raspberry Pi $4 B$ e o sistema operacional Lubuntu. Esta solução é capaz de fornecer condições de acesso adequadas a estudantes cuja situação de vulnerabilidade econômica é acentuada pela obrigatoriedade de isolamento.

Palavras-chave: Ensino remoto, Educação profissionalizante, Raspberry Pi, Linux 


\section{TERMINAL DE ACESSO DE BAIXO CUSTO PARA ENSINO REMOTO}

\section{INTRODUÇÃO}

Durante a suspensão de aulas presenciais de 2020, alternativas digitais foram adotadas para possibilitar algum tipo de ensino remoto. As dificuldades da implementação emergencial de modalidades digitais de comunicação entre professores e alunos incluem a indisponibilidade de acesso a redes de comunicação, pouca ou nenhuma experiência em ambientes virtuais, falta de equipamentos, entre outras. Uma das formas de comunicação que se popularizou foi a videoconferência, que pode ser definida como um meio "um para muitos", que permite o acesso de vários usuários a um acadêmico especialista (LAURILLARD, 2002) Embora esta possa ser realizada via celular, existem vantagens no uso de um computador. Dentre estas vantagens, pode-se citar o uso das demais ferramentas e tecnologias necessárias ao ensino na modalidade remota, a saber: uso de simuladores, uso de softwares para edição de textos e tabelas, etc. As aulas à distância podem ser síncronas ou assíncronas, ou seja, podem ocorrer em tempo real, por meio de videoconferência, ou podem ser gravadas e disponibilizadas aos estudantes. Aulas assíncronas dependem de prazos bem definidos e de estudantes com considerável grau de independência (SLEATOR, 2010). Para estudantes acostumados com a modalidade presencial e que foram obrigados a mudar para a modalidade remota de forma emergencial, contar com este grau de independência pode não ser a melhor forma de garantir um processo de ensino-aprendizagem adequado. Neste sentido, aulas síncronas que possibilitem contato com os docentes tendem a estimular os estudantes. Um estudo realizado por Caton et al. utilizou a quantidade e a qualidade de perguntas feitas por estudantes para comparar o engajamento dos discentes no mesmo curso ofertado nas modalidades presencial e remota. Os autores concluíram que as sessões síncronas com os docentes tiveram aproveitamento melhor do que as aulas presenciais: a qualidade das perguntas realizadas foi maior e os professores despenderam mais tempo e atenção aos estudantes (CATON et al., 2020). Anderson et al. concluíram que a qualidade da interação entre estudantes e professores no ensino remoto é dependente da qualidade de áudio e vídeo e de interações síncronas (ANDERSON et al., 2003). Desta conclusão pode-se associar que a plataforma de hardware utilizada pelo estudante para acessar os recursos do ensino remoto tem impacto na qualidade de seu aprendizado.

Enquanto no ambiente escolar, as condições da sala de aula e dos materiais utilizados pelos docentes eram as mesmas para todos, no ensino remoto, cada estudante fica dependente das condições que tem em seu domicílio. Estudantes cujas famílias são econômica e educacionalmente desfavorecidas sofrem as consequências negativas do ensino remoto de forma desproporcional (CALARCO, 2020). O acesso à internet de qualidade e a equipamentos que facilitem o uso das diversas ferramentas inerentes ao ensino remoto são estruturais na atual situação e podem ser determinantes no que diz respeito ao envolvimento dos estudantes e ao seu desempenho (THURSTON et al., 2021).

Considerando o impacto que uma plataforma de acesso apropriada pode ter no aprendizado dos estudantes durante o ensino remoto, o presente trabalho visa desenvolver um dispositivo que seja adequado ao estudo e possua custo reduzido, buscando atender um maior número de usuários. Para tanto, utilizou-se um kit composto 
de um computador de placa única de tamanho reduzido com os periféricos necessários para a participação em aulas remotas e execução das atividades didáticas.

\section{REVISÃO BIBLIOGRÁFICA}

Com a proibição de aulas presenciais no Brasil em 2020, o sistema educacional viu-se forçado a fazer mudanças e criar estruturas de ensino remoto, uma modalidade para a qual muitas instituições de ensino não estavam plenamente preparadas. Também devido ao curto espaço de tempo em que foi implantado, o sistema como um todo possuía algumas lacunas. Um exemplo é a desigualdade com relação aos meios que possibilitam o acompanhamento das aulas e a realização de exercícios de modo remoto, não só pela falta de acesso à internet, mas como também uma plataforma, como um computador, para tal. Segundo estudo realizado por Dino e Costa (2021), 39\% dos alunos de escolas públicas não têm um computador ou tablet que possa ser usado para estudos. Segundo o IBGE, a maior parte da população brasileira acessa a internet através de celulares (IBGE, 2020).

Para tentar melhorar esta situação dos estudantes, busca-se uma forma barata de participação do ensino remoto com menos limitações. A presente proposta visa disponibilizar um terminal de acesso para aulas remotas com versatilidade e usabilidade similares a um computador e com custo mais acessível do que um celular de entrada. Uma alternativa ao uso de computadores de mesa é a placa Raspberry Pi, desenvolvida pela Raspberry Pi Foundation, uma organização britânica sem fins lucrativos que visa estimular a introdução ao estudo de ciência da computação nas escolas. Esta placa contém os elementos típicos de um computador, como processador, memória, saída para vídeo HDMI, além de portas USB. A proposta deste trabalho consiste em utilizar uma placa Raspberry Pi e uma televisão como monitor. Segundo o IBGE, 96,3\% dos domicílios no Brasil possui pelo menos uma televisão (IBGE, 2020). Além de ter o seu uso mais difundido do que os computadores, o uso da televisão como monitor reduz o custo final da solução proposta.

\subsection{Necessidades de plataformas para ensino remoto}

As atividades típicas de estudantes de ensino regular ou profissionalizante em regime remoto podem compreender: participar de aulas online (gravadas ou ao vivo); fazer pesquisas na internet; ler e escrever textos; e utilizar simuladores. Para essas tarefas, são comumente utilizados computadores, celulares e tablets. Tipicamente, a principal ferramenta para ensino à distância é o computador, seja este portátil ou de mesa (MA et al, 2009). No caso da falta destes, muitos estudantes utilizam o celular para participar das aulas. No entanto, celulares têm várias limitações, como ausência de teclado, tela pequena e o fato de podem apresentar distratores na forma de mensagens e notificações. Além disso, utilizar vários programas simultaneamente na mesma tela não é uma funcionalidade comum em celulares, embora esteja presente em alguns aparelhos. Por exemplo, ao fazer uma pesquisa para um trabalho, o aluno pode precisar alternar constantemente entre um navegador de internet e um editor de texto. Pela ausência de um teclado físico, a digitação também pode ser prejudicada.

Em comparação com os celulares, os tablets têm a vantagem de possuir uma tela maior, mas permanecem as dificuldades de digitação pela falta de teclado físico e mouse, e a dificuldade para utilizar vários programas na mesma tela. Embora não seja comum, é possível utilizar mouse e teclado em tablets e celulares recentes através de recursos bluetooth, USB On-the-Go ou USB 3.0 (ANWARUDDIN E ALI, 2017). O uso de simuladores fica restrito aos disponíveis nas lojas de aplicativos ou que possam ser 
executados no navegador de Internet. Ao se instalar aplicativos de terceiros, pode-se perder a garantia do aparelho (FEJES, et al., 2016).

Utilizando-se um computador de mesa ou portátil, tem-se maior facilidade de se utilizar vários programas simultaneamente, leitura facilitada pela tela maior que a do celular, presença de teclado e mouse que facilitam a execução das atividades didáticas (JUNIOR, 2012) e maior variedade de simuladores. Entretanto, mesmo em residências onde se disponha de computadores, a obrigatoriedade de vários membros da família de estudar ou trabalhar em casa pode levar a uma demanda maior pelo tempo de uso dos dispositivos (PETRUS, 2021). Essa competição pode reduzir o rendimento e desestimular os estudantes.

Neste trabalho, aponta-se a possibilidade de utilização de computadores de placa única como o Raspberry Pi para o ensino remoto. Esta placa possui custo reduzido em relação às outras opções aqui listadas e pode utilizar uma televisão doméstica como monitor, não sendo necessária a compra de um monitor, já que a maioria das residências possui pelo menos uma televisão (IBGE, 2020). A placa é compatível com teclado e mouse USB, o que facilita a digitação e interação, além de ter acesso à rede doméstica, inclusive sem fio (Wi-Fi). Por ser capaz de executar um sistema operacional baseado em Linux, estão disponíveis tanto os simuladores online, que são executados no navegador, quanto os desenvolvidos para sistemas Linux. Essas características proporcionam maior versatilidade ao Raspberry Pi em comparação com celulares e tablets, aproximando suas capacidades às de um computador doméstico. A Figura 1 ilustra a versatilidade dos tipos de equipamentos, ordenando-os do mais barato ao mais caro, e representando sua versatilidade pelo tamanho do item. Dessa forma, percebe-se que o Raspberry Pi é o equipamento mais barato e tem a versatilidade comparável ao dispositivo mais caro, o computador, apesar de ter seu custo inferior ao de um celular de entrada.

Figura 1 - Versatilidade dos dispositivos em função do custo. Cada equipamento está representado com tamanho proporcional à sua versatilidade.

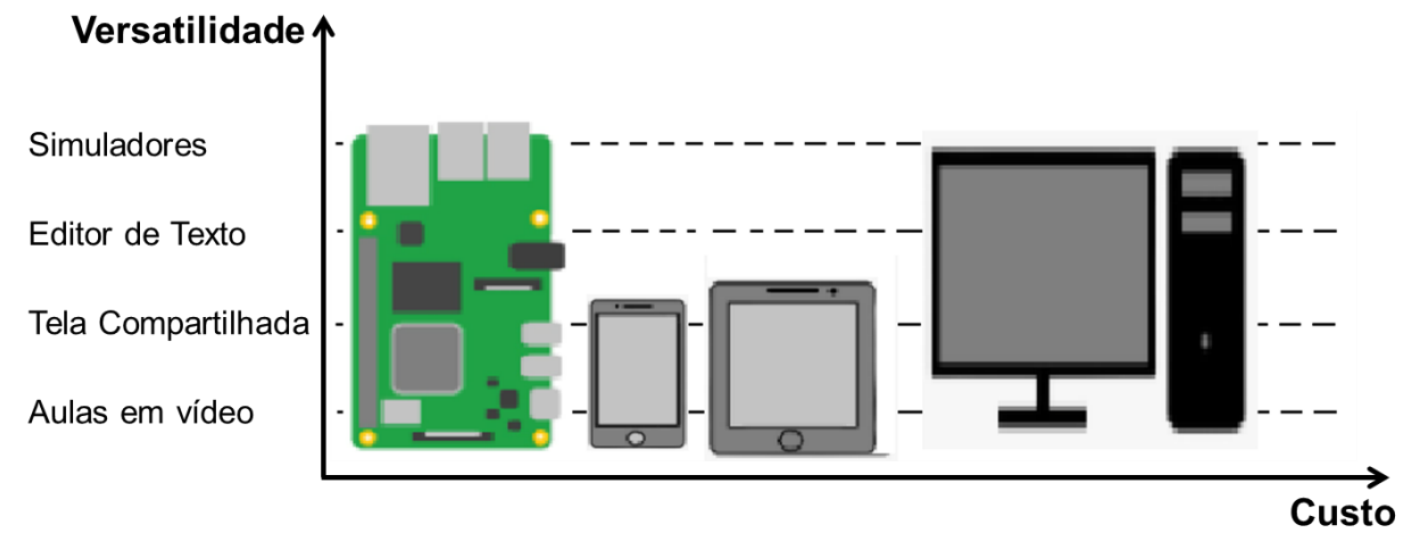

Fonte: elaborada pelos autores.

Com estas considerações, este trabalho considera que um kit composto por uma placa Raspberry $\mathrm{Pi}$, um teclado, um mouse e um televisor pode ser capaz de proporcionar uma melhor experiência ao estudante em ensino remoto em comparação com um celular ou tablet, aproximando-se da versatilidade de um computador. 
Figura 2 - Kit com Raspberry Pi para uso em ensino remoto.

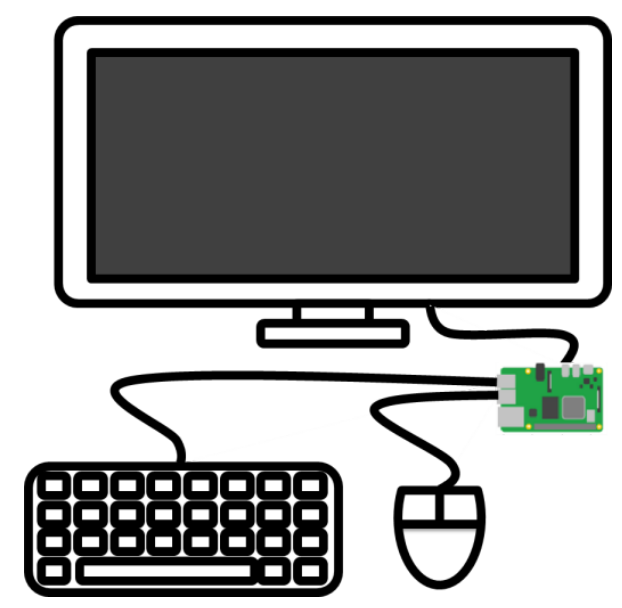

Fonte: elaborada pelos autores.

\subsection{Sistemas operacionais para Raspberry Pi}

A desenvolvedora do Raspberry $\mathrm{Pi}$ possui um sistema operacional próprio chamado Raspbian. Este sistema é baseado na distribuição Linux Debian com adaptações para o uso na linha Raspberry Pi. Este sistema padrão também inclui softwares para uso doméstico e de escritório como navegador de internet, editor de texto, planilhas e apresentações, além de ferramentas de programação para desenvolvedores (RASPBERRYPI, 2021).

Além do Rasbpian, existem vários outros sistemas operacionais compatíveis com a placa que vão desde distribuições Linux sem interface gráfica até sistemas de centro de multimídia (UPTON E HALFACREE, 2014). Um dos sistemas Linux mais populares é o Ubuntu, que se propõe a ter um ambiente de uso mais amigável a usuários pouco experientes, em comparação com distribuições (PAJANKAR, 2020).

Outro sistema é o Lubuntu, uma distribuição baseada em Ubuntu que se caracteriza pelo baixo consumo de memória e processamento. Este sistema foi originalmente desenvolvido para operar em computadores antigos, com pouca memória RAM. Esta característica pode ser vantajosa neste projeto, pois libera recursos para o navegador de internet e chamadas de vídeo, além de permitir tarefas simultâneas (HART-DAVIS, 2017).

\section{METODOLOGIA}

Foram montados kits com diferentes placas e sistemas operacionais a fim de se avaliar o desempenho destes ao se executar tarefas típicas de alunos de ensino remoto. Essas atividades incluíam participação em aulas gravadas e por chamada de vídeo, digitação de textos em editor de texto, navegação na Internet e uso de simuladores. Também foi avaliada a capacidade dos kits de executar vários programas ao mesmo tempo e com várias janelas abertas na mesma tela.

Os testes foram executados por estudantes de curso técnico em automação de nível médio. Os alunos tiveram acesso a duas placas: Raspberry pi 3B e Raspberry pi 4B e fontes de alimentação com tensão de $5 \mathrm{~V}$ e capacidade de fornecimento de corrente de 2 A. A essas placas foram conectados um teclado USB, um mouse USB, uma televisão doméstica com conexão HDMI, um cartão SD de 8 GB e uma rede sem fio Wi-Fi. 
As principais características de cada placa relacionadas com o desempenho são o processador, memória RAM e compatibilidade com rede sem fio. Modelos mais antigos não possuem Wi-Fi integrado, sendo necessário o uso de uma placa de rede USB para acesso sem fio. O modelo 3B possui processador de $1,4 \mathrm{GHz}, 1 \mathrm{~GB}$ de memória e Wi-Fi integrado. O Raspberry $\mathrm{Pi} 4 \mathrm{~B}$ tem um processador de $1,5 \mathrm{GHz}$, Wi-Fi integrado, e foi utilizada a versão com 4 GB de memória. Além das diferenças quantitativas, as versões mais recentes também possuem componentes com tecnologias mais avançadas que contribuem para melhor performance (RASPBERRYPI, 2021).

Os estudantes foram orientados a instalar inicialmente o sistema operacional Raspbian, por ser o recomendado pela desenvolvedora da placa. Posteriormente, foram instalados outros sistemas operacionais, visando encontrar uma configuração que combinasse um bom desempenho e facilidade de uso para usuários pouco experientes. A primeira alternativa considerada foi a distribuição Ubuntu. Em seguida, foi instalada a distribuição Lubuntu. Estes sistemas são gratuitos, baseados em Linux e possuem versões específicas para placas Raspberry.

O sistema foi instalado em um cartão $S D$, visto que a placa não possui armazenamento interno. Após a instalação dos programas básicos no cartão, foram feitos testes de navegação na Internet, edição de texto, reprodução de vídeos e videoconferências. Os testes incluíram ainda videoconferências com vários participantes, com e sem câmera, e observação do efeito da perda de pacotes de dados, a fim de se avaliar subjetivamente a possibilidade de participação em aulas online. Em caso de falta de capacidade do sistema, o aluno pode ser forçado a apenas assistir à aula, mas sem interagir, devido ao atraso e incompletude da comunicação por voz. Além disso, picos de uso dos recursos da placa podem levar à perda de dados de modo a dificultar ou impossibilitar o entendimento e até causar a queda da conexão. A participação em videoconferências e aulas foi feita no Google Meet, por ser a plataforma utilizada na instituição de ensino na qual o projeto foi desenvolvido.

\section{RESULTADOS}

Nos testes realizados, a placa Raspberry $\mathrm{Pi} 4 \mathrm{~B}$ foi a que obteve o melhor desempenho geral. Isso se deveu ao fato de que ela possui um processador mais robusto, Wi-fi e bluetooth embarcados e a maior disponibilidade de memória RAM, possibilitando uma melhor experiência se tratando de multitarefas. Além disso, ela conta com duas entradas USB 3.0 que permitem maior velocidade de transferência de arquivos para uma mídia, como um Pendrive. No entanto, ao se exigir muito do sistema, a temperatura da placa elevava-se, e havia perda de desempenho, causando problemas de comunicação. Por exemplo, em videoconferência utilizando o sistema Raspian e navegador Mozilla Firefox, quando o usuário do Raspberry ligava sua câmera e o áudio, o envio das suas mensagens parecia ser afetado, pois elas passavam a demorar vários segundos para serem enviadas. Além disso, quando um participante da chamada iniciava uma apresentação, o Raspberry perdia conexão. Estes problemas foram corrigidos com a utilização de outro sistema operacional e outro navegador. Com estas adaptações, a placa foi capaz de satisfazer as necessidades de uso levantadas, podendo inclusive gravar vídeos de captura de tela durante os trabalhos.

No modelo $3 \mathrm{~B}$, foi possível executar as principais tarefas desejadas, mas com desempenho pior devido a superaquecimento e falta de memória RAM, principalmente com muitas abas abertas no navegador de internet ou com muitos programas abertos. Isso pode ser um limitador de uso para usuários pouco experientes, pois é necessário planejar as atividades e gerenciar os recursos do sistema. 
No quesito de software, o Lubuntu foi escolhido dentre outros dois concorrentes, Ubuntu e Raspbian. Sua otimização de recursos, compatibilidade com a arquitetura do hardware da placa, capacidade para multitarefas e usabilidade foram pontos determinantes. A placa foi testada para assegurar se seria possível um uso fluido e estável com navegadores, simuladores e ferramentas de videoconferência diferentes. Adicionalmente, observou-se que o navegador de internet Chromium apresentou melhor desempenho.

Os autores fizeram videoconferências, sendo que um dos alunos participou utilizando o Raspberry. Foram avaliadas as condições de comunicação em diferentes situações, e cada participante atribuiu notas para estas, sendo 1 a pior nota, e 5 a melhor. Alguns participantes participaram utilizando computadores, outros via celular, e um a partir do Raspberry Pi. Foi analisada a experiência em nove itens ou situações considerados relevantes para a comunicação por videoconferência: qualidade de comunicação com todas as câmeras ativas; apenas o professor com câmera ativa; professor compartilhando tela ou apresentando um vídeo com câmera desligada; professor compartilhando tela ou apresentando um vídeo apenas com sua câmera ligada; professor compartilhando tela ou apresentando um vídeo com todas as câmeras ligadas; qualidade geral do vídeo; qualidade geral do áudio; inteligibilidade da voz para comunicação (considerando atrasos e cortes no áudio) e uso da ferramenta de comunicação por mensagens de texto (chat). A Figura 3 apresenta a comparação das notas atribuídas de maneira independente pelos autores nos primeiros testes e na configuração final obtida. A videoconferência inicial foi feita em Ubuntu e Mozilla Firefox. A última chamada de teste foi feita com Lubuntu e Chromium. Nota-se que quase todas as atividades realizadas foram melhor executadas após as trocas de sistema operacional e navegador. Com essas alterações, a experiência do usuário com Raspberry Pi foi melhor, e os outros usuários tiveram mais facilidade em se comunicar com este.

Figura 3 - Comparação entre videoconferências utilizando Lubuntu/Chromium e Ubuntu/Firefox.

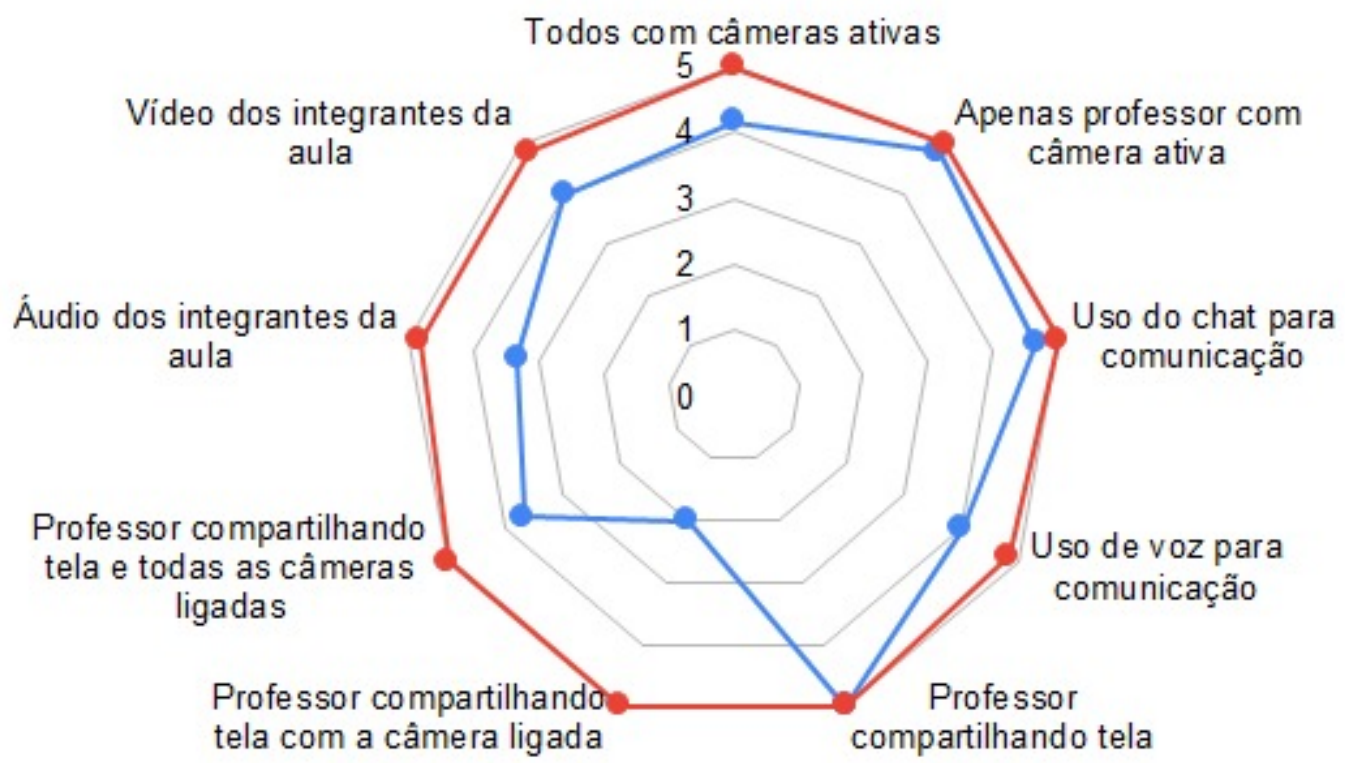

Fonte: elaborada pelos autores. 
O projeto mostrou-se uma solução satisfatória para o problema, sendo uma opção viável que poderia substituir celulares, tablets e notebooks de entrada no ensino à distância. Os pontos que mais se destacam são não apenas o maior conforto e praticidade na realização de trabalhos acadêmicos e a maior compatibilidade com softwares de simulação em navegadores, como também a possibilidade de realizar mais de uma tarefa enquanto assiste a uma aula, como digitar um texto ou fazer uma pesquisa na Internet. Um ponto negativo é a ausência de entrada de microfone na placa, levando à necessidade do uso de algum adaptador ou microfone USB, caso o estudante precise participar de uma chamada com voz, por exemplo.

\section{CONSIDERAÇÕES FINAIS}

O kit foi preparado para ser usado como terminal de acesso à internet por alunos que não dispõem de computador. Este kit pode ser conectado a uma televisão com entrada HDMI. Dentre as vantagens do desenvolvimento deste kit cita-se o baixo custo da solução proposta, menor do que o custo de um telefone celular de entrada e cerca de um terço do custo de computadores comerciais. Esta solução é capaz de fornecer condições de acesso adequadas a estudantes cuja situação de vulnerabilidade econômica é acentuada pela obrigatoriedade de isolamento. Desta forma, o projeto busca reduzir a desigualdade de condições entre os estudantes, a fim de que o processo de aprendizado seja menos prejudicado. Os discentes envolvidos no projeto tiveram experiência com instalação e configuração de sistemas Linux e rede de comunicação de computadores. Os estudantes executaram testes em diferentes combinações de softwares e fizeram análises do desempenho do sistema em diversas condições para determinar uma combinação de equipamentos e softwares capaz de permitir velocidade e facilidade de uso suficientes para alunos com pouca experiência com aulas virtuais e em sistema Linux. Entre as combinações testadas, a melhor foi o kit contendo uma placa Raspberry Pi 4B com 4 GB de memória RAM e o sistema operacional Lubuntu, rodando o navegador Chromium.

\section{REFERÊNCIAS}

ANDERSON, R.; BEAVERS, J.; VANDEGRIFT, T.; VIDEON, F. Videoconferencing and presentation support for synchronous distance learning. In: 33rd Annual Frontiers in Education, 2003, Westminster, CO, EUA. Disponível em: https://ieeexplore.ieee.org/abstract/document/1264746. Acesso em: 19/05/2021.

ANWARUDDIN, M.; ALI, M. A. A Review On Raspberry Pi Based Industrial Process Monitoring And Control Using Modbus Protocol. IJITR INTERNATIONAL JOURNAL OF INNOVATIVE TECHNOLOGY AND RESEARCH Volume, n. 5, p. 5483-5486, 2017

CALARCO, J. M. Avoiding us versus them: how schools dependence on privileged helicopter parents enforcement of rules. American Sociological Review, v. 85, n. 2, p. 223-246, 2020.

CATON, J. B; CHUNG, S.; ADENIJI, N.; HOM, J.; BRAR, K.; GALLANT, A.; BRYANT, M.; HAIN, A.; BASAVIAH, P.; HOSAMANI, P. Student engagement in the online classroom: comparing preclinical medical student question-asking behaviors in a videoconference versus in-person learning environment. Faseb Bio Advances, v. 3, n. 2, p. 110-117, 2020. 
DINO, L. A.; COSTA, D. Uso da Internet por crianças e adolescentes no Brasil: dinâmicas e desafios. RE@ D-Revista de Educação a Distância e Elearning, v. 4, n. 1, p. 25-41, 2021.

DORN, E.; HANCOCK, B.; SARAKATSANNIS, J; VIRULEG, E. COVID-19 and student learning in the United States: the hurt could last a lifetime.

FEJES, F.; KATONA, R.; PÜSÖK, L. Multipath strategies and solutions in multihomed mobile environments. In: 2016 7th IEEE International Conference on Cognitive Infocommunications (CoglnfoCom). IEEE, 2016. p. 000079-000084.

HART-DAVIS, G. Choosing Operating Systems for Raspberry Pi. In: Deploying Raspberry Pi in the Classroom. Apress, Berkeley, CA, 2017. p. 55-69.

Instituto Brasileiro de Geografia e Estatística. Pesquisa Nacional por Amostra de Domicílios Contínua - PNAD Contínua, tema suplementar Tecnologia da Informação e Comunicação - TIC nos aspectos de acesso à Internet e à televisão e posse de telefone móvel celular para uso pessoal. 2019. Disponível em: https://biblioteca.ibge.gov.br/visualizacao/livros/liv101705_informativo.pdf. Acesso em: 18 mai. 2020.

JUNIOR, J. B. B. Do computador ao tablet: Vantagens pedagógicas na utilização de dispositivos móveis na educação/from computer to tablet: Advantages in the pedagogical use of mobile devices in education. Revista educaonline, v. 6, n. 1, p. 125-149, 2012.

LAURILLARD, D. Rethinking university teaching: a framework for the effective use of educational technology. 2. ed. Londres: Taylor \& Francis, 2002.

MA, X. D.; TAYLOR, C. J.; JOYCE, M. J. An interactive PC-based electrical power system simulator for engineering education. In: Proceedings of the 5th International CDIO Conference, Singapore Polytechnic, Singapore. 2009. p. 7-10.

PAJANKAR, A. Raspberry $\mathrm{Pi}$ Computer Vision Programming: Design and implement computer vision applications with Raspberry Pi, OpenCV, and Python 3. Packt Publishing Ltd, 2020.

PETRUS, J. S. R. et al. Simulação de desigualdades educacionais acirradas pela pandemia da Covid-19. Revista Brasileira de Avaliação, v. 10, n. 1, p. 0-0, 2021.

RaspberryPi. Raspberry Pi computers and microcontrollers. Disponível em: https://www.raspberrypi.org/products/ Acesso em 18/05/2021.

SLEATOR, R. D.; The evolution of learning background, blends and blackboard. Science Progress, v. 93, n. 3, p. 319-334, 2010.

THURSTON, D.; RENZULLI, L.; MURRAY, B.; GARZA, A. N.; PEREZ, L. Remote or removed: predicting successful engagement with online learning during COVID-19. Socius: sociological research for a dynamic world. v. 7, p. 1-15, 2021.

UPTON, E.; HALFACREE, Gareth. Raspberry Pi user guide. John Wiley \& Sons, 2014. 


\title{
LOW COST ACCESS TERMINAL FOR REMOTE LEARNING
}

\begin{abstract}
Due to the suspension of classroom courses in Brazil during 2020, digital alternatives were adopted to enable remote education. One of the forms of communication that became popular was video conferencing. The present project aims to offer a solution to one of the difficulties of remote activities: the lack of an adequate device for the study. For this, we used a kit composed of a single board computer of reduced size with the necessary peripherals for participation in remote classes and execution of didactic activities. The kit was prepared to be used as an internet access terminal by students who do not have a computer, and it can be connected to a television with HDMI input. This allows a lower cost in comparison with commercial computers. Different cards and operating systems were tested, and satisfactory results were obtained for internet browsing and video calls with the Raspberry Pi 4B card and the Lubuntu operating system. This solution is able to provide adequate access conditions for students whose economic vulnerability is accentuated by the requirement of isolation.
\end{abstract}

Keyword: Remote education, Vocational education, Raspberry Pi, Linux 